\title{
¿Es la jubilación un buen momento para aprender informática?
}

\author{
Cristina Vilaplana Prieto \\ Departamento de Fundamentos de Análisis Económico. Universidad de Murcia, España \\ cvilaplana@um.es
}

\begin{abstract}
The aim of this paper is to disentangle the characteristics of older people who know computer science and test if older people consider retirement as a good moment for learning this issue. We use data from the Survey of Living Conditions of Older People (2010) carried out by the IMSERso to individuals aged 65 and older. Available information allow us to differentiate among three categories of users: (1) those who knew computer science before getting retired, pre-users $(6.79 \%),(2)$ those who learnt it after retirement, post-users $(2.76 \%)$ and (3) those who have never expressed interest in it, never-users (90.45\%). As compared to never-users, people qualified as pre-users or post-users are to a large extent male, aged 65-69, with high school or college education, income higher than $900 € / \mathrm{month}$ and, they usually combine this activity with others such as tourism, sports and volunteerism. To determine which variables affect the probability of becoming post-user we estimate a bivariate probit model with sample selection. The probability of becoming post-user increases by $151 \%$ for those who consider that retirement is "an opportunity for pursuing your particular hobbies" and it increases by $71 \%$ for those who report that their main fear for the future is to loose memory. However, living in a municipality with less than 5,000 inhabitants or being a housekeeper during working life decreases this probability. On the other hand, the well suited moment for becoming familiar with computer science is just after retirement because the probability of becoming post-user increases by $245 \%$ for the cohort aged $65-59$ years.
\end{abstract}

Keywords: older people, computer science, internet, bivariate probit model, sample selection

\section{Resumen}

El objetivo de este trabajo es conocer las características de las personas mayores que saben informática y contrastar si las personas mayores consideran que la etapa de la jubilación es un buen momento para aprender dicha materia. Se utilizan datos de la Encuesta de Condiciones de Vida de las Personas Mayores (2010) realizada por el IMSERso a personas de 65 y más años. La información dispone tres categorías de usuarios: 1) personas que sabían informática antes de la jubilación, usuarios-pre (6,79 \%), 2) los que la aprendieron después de la jubilación, usuarios-post (2,76 \%) y 3 ) los que nunca han mostrado interés, no usuarios (90,45\%). En comparación con los no usuarios, los usuarios pre o post suelen ser en mayor medida hombres, de 65-69 años, con estudios secundarios o superiores, con ingresos superiores a $900 € /$ mes y combinan la informática con otras actividades como turismo, deporte y voluntariado. Para determinar qué variables influyen en la probabilidad de convertirse en un usuario-post se estima un modelo probit bivariante con selección muestral. La probabilidad de ser usuario-post aumenta un $151 \%$ entre los que consideran que la jubilación es «una oportunidad para dedicar su tiempo a lo que quiere» y aumenta un $71 \%$ entre aquellos que declaran que su principal miedo de cara el futuro es el de perder la memoria. En cambio, los que viven en municipios de menos de 5000 habitantes o los que se han dedicado a las labores del hogar demuestran una menor probabilidad de convertirse en usuarios. Por otra parte, el momento idóneo para familiarizarse con la informática es justo después de la jubilación, ya que la probabilidad de ser usuario-post aumenta un $245 \%$ para la cohorte de 65-69 años.

Palabras clave: personas mayores, informática, Internet, modelo probit bivariante, selección muestral 


\section{Datos y estadísticos descriptivos}

Los datos utilizados en este trabajo proceden de la Encuesta de Condiciones de Vida de las Personas Mayores, realizada por el IMSERSO en el año 2010. Se trata de una encuesta realizada mediante entrevista telefónica a una muestra de 2535 personas de 65 y más años, residentes en España (excepto Ceuta y

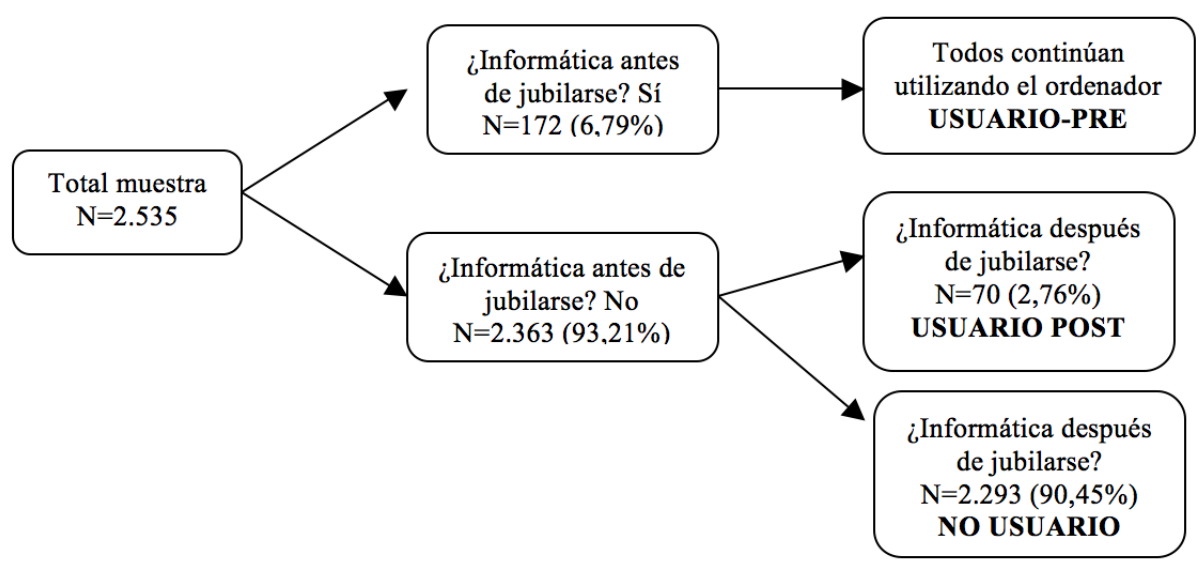

Figura 1. Distribución de la muestra

Melilla). La muestra se selec-

cionó mediante muestreo aleatorio simple a partir de listín telefónico, utilizando como variables de cuota la edad, el sexo, el tamaño de municipio y la comunidad autónoma. Las personas entrevistadas respondieron a un cuestionario telefónico estructurado, compuesto por preguntas cerradas y organizado en bloques temáticos: 1) características sociodemográficas, 2) vivienda, 3) uso del tiempo, 4) estado de salud, 5) asistencia socio-sanitaria y dependencia, 6) renta y consumo, 7) ciudadanía y participación social y 8) envejecimiento como experiencia vital.

Dentro del módulo de uso del tiempo se pregunta a los entrevistados si utilizan el ordenador (con o sin conexión a internet), respondiendo afirmativamente el 9,55\%, que en términos poblacionales supone 762311 usuarios. También se les pregunta si, a partir de la jubilación, han iniciado alguna actividad nueva que no hubieran realizado antes. Entre las opciones disponibles se encuentra la de informática, que es respondida por el 2,76 \% de los entrevistados. Estas dos preguntas permiten distinguir entre aquellas personas que utilizaban el ordenador antes de jubilarse y los que se han familiarizado con la informática una vez jubilados.

Con el objeto de simplificar la notación a utilizar se definen las siguientes variables: 1) usuario-pre: indicador binario que toma el valor 1 si la persona utilizaba el ordenador antes de jubilarse; 2) usuario-post: indicador binario que toma el valor 1 si la persona ha aprendido informática después de jubilarse; 3) no usuario: indicador binario que toma el

valor 1 si la persona no utiliza (ni antes ni ahora) el ordenador. La figura 1 describe la composición de la muestra en términos de estas variables. Es necesario destacar que todos aquellos que son usuariospre han manifestado que continúan utilizando el ordenador una vez jubilados.

La comparación de usuarios frente a no usuarios revela características sociodemográficas diferenciadas. Entre los usuarios, el porcentaje de hombres es superior (58,35\% frente a 40,56 \% para no usuarios), mayor concentración en la cohorte de 65-69 años (53,42\% frente a $21,44 \%)$, no suelen vivir en municipios de menos de 5000 habitantes $(5,84 \%$ frente a $22,80 \%)$, mayor porcentaje con ingresos superiores a $900 € /$ mes $(45,89 \%$ frente a $12,35 \%)$. Los usuarios suelen participar más en otras actividades: deporte $(42,49 \%$ frente a 25,12 $\%)$, turismo $(20,09 \%$ frente a $7,50 \%)$ y voluntariado $(16,88 \%$ frente a $8,37 \%)$.

El nivel educativo es sustancialmente diferente entre ambos grupos: entre los usuarios, el 61,58\% tiene estudios secundarios o superiores, mientras que el 75,97 \% de los no usuarios no ha finalizado los estudios primarios. Por otra parte, el 31,59\% de los usuarios tiene conocimientos de otro idioma (inglés, francés, alemán o italiano) frente a solo el $3,74 \%$ de los no usuarios.

\section{Modelo}

Consideramos que el hecho de ser o no usuariopre, usuario-post o no usuario es consecuencia de las decisiones que toma el individuo a lo largo de 
Tabla 1. Estadísticos descriptivos

\begin{tabular}{|c|c|c|c|}
\hline & \multicolumn{3}{|c|}{ Usuario } \\
\hline & No & post & pre \\
\hline $\mathrm{N}$ & 2,293 & 70 & 172 \\
\hline \multicolumn{4}{|l|}{ Sexo } \\
\hline Hombre & 40,56 & 59,39 & 58,35 \\
\hline Mujer & 59,44 & 40,61 & 41,65 \\
\hline \multicolumn{4}{|l|}{ Edad } \\
\hline $65-69$ & 21,44 & 42,77 & 53,42 \\
\hline $70-74$ & 25,96 & 30,44 & 24,73 \\
\hline $75-79$ & 23,25 & 13,38 & 12,77 \\
\hline 80 y más & 29,35 & 13,41 & 9,07 \\
\hline \multicolumn{4}{|l|}{ Educación } \\
\hline Sin estudios & 75,97 & 38,69 & 14,46 \\
\hline Primarios & 15,23 & 8,02 & 22,41 \\
\hline Secundarios & 3,89 & 38,06 & 41,90 \\
\hline Superiores & 1,44 & 14,15 & 19,68 \\
\hline $\begin{array}{l}\text { Sabe algún idioma ex- } \\
\text { tranjero }\end{array}$ & 3,74 & 32,23 & 31,59 \\
\hline \multicolumn{4}{|l|}{ Ingresos mensuales } \\
\hline Menos de $300 €$ & 18,12 & 21,25 & 13,60 \\
\hline Entre $300 €$ y $600 €$ & 36,01 & 6,36 & 5,78 \\
\hline Entre $600 €$ y $900 €$ & 20,89 & 33,60 & 23,90 \\
\hline Entre $900 €$ y $1.200 €$ & 7,02 & 6,75 & 20,08 \\
\hline Más de $1,200 €$ & 5,33 & 23,79 & 25,81 \\
\hline \multicolumn{4}{|l|}{ Tamaño del municipio } \\
\hline Hasta 5000 hab. & 22,80 & 1,44 & 5,84 \\
\hline 5001-10.000 hab. & 9,17 & 3,58 & 5,47 \\
\hline 10001-20000 hab. & 10,13 & 24,21 & 9,06 \\
\hline 20001-100000 hab. & 22,57 & 21,07 & 15,70 \\
\hline Más de 100000 hab. & 35,34 & 49,70 & 63,93 \\
\hline \multicolumn{4}{|l|}{ Forma de convivencia } \\
\hline Solo & 16,54 & 3,41 & 5,90 \\
\hline Cónyuge e hijos & 15,15 & 41,37 & 41,32 \\
\hline Cónyuge (sin hijos) & 41,45 & 40,42 & 37,45 \\
\hline Vive con hijos & 18,57 & 5,83 & 5,82 \\
\hline $\begin{array}{l}\text { Vive solo (pero no le } \\
\text { gusta) }\end{array}$ & 11,86 & 0,74 & 2,41 \\
\hline
\end{tabular}

\begin{tabular}{|c|c|c|c|}
\hline & \multicolumn{3}{|c|}{ Usuario } \\
\hline & no & post & pre \\
\hline \multicolumn{4}{|c|}{ Durante su vida laboral, trabaja como } \\
\hline Asalariado & 42,35 & 69,51 & 68,77 \\
\hline Empresario & 17,91 & 7,38 & 13,74 \\
\hline Activ. domésticas & 38,60 & 22,33 & 16,88 \\
\hline \multicolumn{4}{|l|}{ Situación actual } \\
\hline Todavía trabaja & 0,60 & 1,90 & 1,21 \\
\hline $\begin{array}{l}\text { Jubilado (trabajó an- } \\
\text { tes) }\end{array}$ & 56,88 & 73,16 & 80,26 \\
\hline $\begin{array}{l}\text { Pensionista (antes ama } \\
\text { de casa) }\end{array}$ & 16,32 & 2,61 & 1,56 \\
\hline $\begin{array}{l}\text { Actividades domésti- } \\
\text { cas }\end{array}$ & 26,04 & 22,33 & 16,78 \\
\hline \multicolumn{4}{|c|}{ Nuevas actividades con la jubilación } \\
\hline Informática & 0,00 & 100,00 & 24,40 \\
\hline Turismo & 7,50 & 23,36 & 20,09 \\
\hline Formación, clases & 0,51 & 0,00 & 1,57 \\
\hline Deporte & 25,12 & 41,51 & 42,49 \\
\hline Manualidades & 11,70 & 11,69 & 15,64 \\
\hline $\begin{array}{l}\text { Cultivar un huerto o } \\
\text { jardín }\end{array}$ & 5,18 & 5,23 & 3,78 \\
\hline $\begin{array}{l}\text { Hacer amigos / salir } \\
\text { con amigos }\end{array}$ & 6,36 & 7,38 & 6,08 \\
\hline $\begin{array}{l}\text { Participar en alguna } \\
\text { asociación }\end{array}$ & 2,52 & 3,72 & 1,79 \\
\hline Voluntariado & 8,37 & 23,21 & 16,03 \\
\hline \multicolumn{4}{|l|}{ Cómo vive la jubilación } \\
\hline $\begin{array}{l}\text { Tiempo para lo que } \\
\text { quiere }\end{array}$ & 15,39 & 27,34 & 29,45 \\
\hline $\begin{array}{l}\text { Su vida ha perdido } \\
\text { sentido }\end{array}$ & 11,93 & 16,42 & 12,49 \\
\hline \multicolumn{4}{|c|}{ A qué tiene miedo en el futuro } \\
\hline A la pérdida de salud & 51,96 & 54,93 & 53,92 \\
\hline A la soledad & 30,52 & 32,51 & 29,00 \\
\hline $\begin{array}{l}\text { A la pérdida de me- } \\
\text { moria }\end{array}$ & 7,35 & 3,57 & 3,25 \\
\hline $\begin{array}{l}\text { A no poder valerse } \\
\text { por sí mismo }\end{array}$ & 36,54 & 40,15 & 38,19 \\
\hline $\begin{array}{l}\text { No tiene enferme- } \\
\text { dades crónicas }\end{array}$ & 13,58 & 19,44 & 19,77 \\
\hline
\end{tabular}


un periodo de tiempo. Por consiguiente, podemos construir un modelo secuencial en dos periodos: el primer periodo corresponde a la etapa previa a la jubilación y el segundo periodo a la etapa posterior a la jubilación. Se va a utilizar un modelo probit bivariante con selección muestral (van de Ven y van Praag, 1981; Greene, 1993). La ventaja de este modelo es que permite que exista correlación entre las variables inobservables que afectan la decisión de no ser usuario en el primer periodo, con las variables inobservables que afectan a la probabilidad de ser usuario en el segundo periodo. Por consiguiente, para cada individuo $\mathrm{i}, \mathrm{i}=1, \ldots, \mathrm{N}$ la utilidad en cada momento del tiempo $t, t=1,2$ se puede expresar como la suma de dos componentes:

$$
\begin{aligned}
& t=1 \Rightarrow U_{i j 1}=\beta^{\prime}{ }_{j 1} X_{i j 1}+\varepsilon_{i j 1} \quad j=\text { usuario-pre,no usuario } \\
& t=2 \Rightarrow U_{i j 2}=\beta^{\prime}{ }_{j 2} X_{i j 2}+\varepsilon_{i j 2} \quad j=\text { usuario-post, no usuario }
\end{aligned}
$$

donde $\mathrm{X}_{\mathrm{ij1}}, \mathrm{X}_{\mathrm{ij} 2}$ se refieren al componente observado (características socioeconómicas del individuo $\mathrm{y}$ variables del entorno) mientras que $\varepsilon_{\mathrm{ij} 1}, \varepsilon_{\mathrm{ij} 2}$ son términos de error aleatorios e inobservables. Suponemos que los términos de error se distribuyen según una normal bivariante $\varepsilon_{\mathrm{ij} 1}, \varepsilon_{\mathrm{ij} 2} \sim \mathrm{N}(0,0,1,1, \rho)$. Aunque la utilidad total es una variable inobservable, las elecciones del individuo sí que son observables. En el periodo 1, un individuo decidirá convertirse en usuario-pre cuando dicha elección maximice su utilidad en dicho periodo. Por la misma razón, un individuo decidirá convertirse en usuario-post cuando, condicional a no haber utilizado los ordenadores durante el periodo previo a la jubilación, decida familiarizarse con la informática en un momento posterior a su jubilación.

$$
\begin{aligned}
\text { Usuario }- \text { pre }_{i} & =\left\{\begin{array}{l}
1 \text { si } U_{i 1}>0 \\
0 \text { en } \text { otro } \text { caso }
\end{array}\right. \\
\text { Usuario }- \text { post }_{i} & =\left\{\begin{array}{l}
1 \text { si } U_{i 2}>0 \\
0 \text { en otro caso }
\end{array}\right.
\end{aligned}
$$

La función de verosimilitud correspondiente es:

$$
\begin{aligned}
L= & \sum \ln \Phi_{2}\left[-\beta_{1}{ }^{\prime} X_{i 1}, \beta_{2}{ }^{\prime} X_{i 2}, \rho\right]+ \\
& \sum_{y_{i 1}=0, y_{i 2}=0} \ln \Phi_{2}\left[-\beta_{1}{ }^{\prime} X_{i 1},-\beta_{2}{ }^{\prime} X_{i 2}, \rho\right]+\sum_{y_{i 1}=1} \ln \Phi\left[\beta_{1}{ }^{\prime} X_{i 1}\right]
\end{aligned}
$$

Dentro de las variables explicativas de la decisión de ser usuario-pre $\mathrm{X}_{\mathrm{i} 1}$, incluimos: género, nivel educativo, saber idiomas, tamaño de municipio de residencia y relación con la actividad económica antes de jubilarse.

Entre las variables explicativas de la ecuación de usuario-post $\mathrm{X}_{\mathrm{i} 2}$, también incluimos género, nivel educativo, saber idiomas y tamaño del municipio de residencia, pero además, y actuando como restricciones de exclusión, nivel de ingresos (después de la jubilación), la forma que tienen las personas de afrontar la jubilación, los miedos respecto al futuro, el hecho de vivir solo y el estar bien de salud.

No incluimos las variables relativas a la realización de otras actividades para evitar un problema de causalidad inversa. Por razones de espacio, no se muestran los coeficientes estimados, sino simplemente las probabilidades predichas. La tabla 2 muestra la probabilidad de convertirse en usuariopost para un determinado caso base, así como las variaciones en dicha probabilidad ante cambios en las variables explicativas.

La probabilidad de convertirse en usuario-post aumenta 245,82\% si se tiene entre 65 y 69 años, confirmando que el momento idóneo para familiarizarse con la informática es justo después de la jubilación. No obstante, la edad no es el único factor relevante. Aquellas personas que consideran que la jubilación es «una oportunidad para dedicar su tiempo a lo que quiere» manifiestan un incremento del 151,23 \% en la probabilidad de convertirse en usuarios-post. En este mismo sentido, el miedo a perder la memoria aumenta en un 71,59 \% la probabilidad de convertirse en usuario-post.

En relación al nivel de estudios, las personas que sólo terminaron la educación secundaria son las que manifiestan un mayor interés por la informática una vez jubiladas $(751,41 \%)$. Merece la pena destacar que las personas con estudios superiores no demuestran un mayor interés por las nuevas tecnologías durante la jubilación, bien porque no tuvieron que utilizarlas durante su vida laboral o porque nunca les han resultado lo suficientemente atractivas.

En relación a la actividad económica previa a la jubilación, si durante la etapa laboral las personas 
Tabla 2. Probabilidades predichas de convertirse en usuario-post

\begin{tabular}{|c|c|c|c|}
\hline Prob caso base $=0.0082$ & $\begin{array}{l}\text { Probabilida } \\
\text { predicha }\end{array}$ & $\mathrm{p}$-value & $\begin{array}{l}\text { Incremento } \\
\text { respecto caso } \\
\text { base }(\%)\end{array}$ \\
\hline Hombre & 0,0102 & 0,632 & 21,81 \\
\hline \multicolumn{4}{|l|}{ Edad } \\
\hline $65-69$ & 0,0284 & $0,049^{\star *}$ & 245,82 \\
\hline $70-74$ & 0,0188 & 0,182 & 128,08 \\
\hline $75-79$ & 0,0073 & 0,863 & $-11,33$ \\
\hline \multicolumn{4}{|l|}{ Tamaño del municipio } \\
\hline Hasta 5000 hab. & $-0,0008$ & $0,015^{\star *}$ & $-109,43$ \\
\hline 5001-10000 hab. & 0,0057 & 0,540 & $-31,99$ \\
\hline 10001-20000 hab. & 0,0324 & $0,017^{\star \star}$ & 285,44 \\
\hline 20001-100000 hab. & 0,0094 & 0,820 & 12,06 \\
\hline \multicolumn{4}{|l|}{ Nivel de ingresos } \\
\hline Menos de $300 €$ & 0,0097 & 0,814 & 18,19 \\
\hline Entre $300 €$ y $600 €$ & 0,0018 & 0,138 & $-77,73$ \\
\hline Entre $600 €$ y $900 €$ & 0,0117 & 0,474 & 42,76 \\
\hline Entre $900 €$ y $1200 €$ & 0,0033 & 0,113 & $-60,11$ \\
\hline \multicolumn{4}{|l|}{ Nivel de educación } \\
\hline Primarios & 0,0104 & 0,660 & 26,41 \\
\hline Secundarios & 0,0700 & $0,007^{\star * *}$ & 751,41 \\
\hline Superiores & 0,0337 & 0,231 & 309,92 \\
\hline Sabe idiomas & 0,0221 & 0,014 & 169,05 \\
\hline \multicolumn{4}{|l|}{ Antes de la jubilación } \\
\hline Trabajaba como asalariado & 0.0190 & $0.032^{\star *}$ & 126.86 \\
\hline Trabajaba como empresario & 0.0185 & $0.021^{\star *}$ & 120.11 \\
\hline \multicolumn{4}{|l|}{ Cómo vive la jubilación } \\
\hline Tiempo para lo que quiere & 0.0211 & $0,027^{\star *}$ & 151,23 \\
\hline Su vida ha perdido sentido & 0,0064 & $0,036^{* *}$ & $-21,67$ \\
\hline Miedo a perder la memoria & 0,0141 & $0,043^{* *}$ & 71,59 \\
\hline Vive solo & 0,0020 & $0,080^{*}$ & $-75,59$ \\
\hline Estar bien de salud & 0,0087 & 0,898 & 5,32 \\
\hline
\end{tabular}

${ }^{* * *}$ : significativo al $1 \%$; ${ }^{* *}$ : significativo al $5 \%$; $^{*}$ : significativo al $\left.10 \%\right)$

Caso base: mujer, de 80 y más años, sin estudios, dedicada a labores domésticas 
que trabajaron como asalariados o empresarios no tuvieron contacto con la informática, es un $121 \%$ más probable que comiencen a hacerlo después de jubilados en comparación con las personas que estuvieron dedicadas a las labores del hogar exclusivamente. Por último, el hecho de vivir en un municipio de menos de 5000 habitantes o de vivir solo disminuye dicha probabilidad en un $109,43 \%$ y $75,59 \%$, respectivamente.

\section{Conclusiones}

Las conclusiones finales del trabajo son las siguientes. Primero, el porcentaje de usuarios entre las personas mayores es muy bajo (inferior al 10\%). Segundo, la mayoría de los usuarios comenzaron a serlo antes de jubilarse $y$, una vez retirados, to- dos han seguido utilizando el ordenador. Tercero, la probabilidad de convertirse en un nuevo usuario aumenta entre aquellos que interpretan la jubilación de forma positiva y fructífera. Por último, se recomienda que las acciones formativas o de difusión de nuevas tecnologías puedan: 1) ampliar su cobertura geográfica dirigiéndose a municipios pequeños; 2) realizar programas formativos dirigidos a públicos distintos (personas con estudios superiores, personas que han estado dedicadas a las labores del hogar); y 3) dirigirse principalmente a un público de entre 65 y 69 años, puesto que, aunque hay usuarios de más de 80 años, los primeros años después de la jubilación son los más decisivos a la hora de convertirse en un nuevo usuario.

\section{Referencias}

Greene, W. (1993). Econometric analysis. Nueva York: MacMillan Publishing Company.

Van de Ven, W. P. y Van Praag, B. M. (1981). The demand for deductibles in private health insurance: a probit model with sample selection. Journal of Econometrics 17, 229-252. 